\title{
Revision total knee arthroplasty: the end of the allograft era?
}

\author{
Sébastien Parratte ${ }^{1} \cdot$ Matthew P. Abdel $^{2} \cdot$ Alexandre Lunebourg $^{1} \cdot$ \\ Nicolaas Budhiparama ${ }^{3}$ David G. Lewallen ${ }^{2} \cdot$ Arlen D. Hanssen ${ }^{2}$. \\ Jean-Noël Argenson ${ }^{1}$
}

Received: 18 February 2015/ Accepted: 27 February 2015/Published online: 10 March 2015

(C) Springer-Verlag France 2015

Total knee arthroplasty (TKA) is highly successful, with an exponential increase expected in the near future $[1,2]$. More importantly, a fivefold increase in the number of revision TKAs is expected by 2030 [1,2]. In the latest studies, the top seven reasons for revision TKA include aseptic loosening $(23.1 \%)$, infection $(18.4 \%)$, polyethylene wear $(18.1 \%)$, instability $(17.7 \%)$, pain/stiffness $(9.3 \%)$, osteolysis $(4.5 \%)$, and malposition/misalignment $(2.9 \%)[1,3]$. With modern implants, constraint can be effectively managed [4]. However, one of the remaining challenges in revision TKA is the management of severe

Sébastien Parratte

sebparratte@gmail.com; sebastien@parratte.fr

Matthew P. Abdel

abdel.matthew@mayo.edu

Alexandre Lunebourg

alexandre.Lunebourg@gmail.com

Nicolaas Budhiparama

ncbjr@yahoo.com

David G. Lewallen

lewallen.david@mayo.edu

Arlen D. Hanssen

Hanssen.arlen@mayo.edu

Jean-Noël Argenson

Jean-noel.ARGENSON@ap-hm.fr

1 Department of Orthopedics and Traumatology, Institute for Locomotion, Hôpital St. Marguerite, 270 Boulevard Sainte Marguerite, BP 29, 13274 Marseille, France

2 Department of Orthopedic Surgery, Mayo Clinic, 200 First Street S.W., Rochester, MN 55905, USA

3 Nicolaas Institute of Constructive Orthopaedics Research and Education Foundation for Arthroplasty and Sports Medicine, Medistra Hospital, Jl. Jend. Gatot Subroto Kav. 59, Jakarta 12950, Indonesia bone loss [4]. Traditionally, allografts have been widely utilized to manage bone loss, with a significant failure rate at only mid-term follow-up [4]. New techniques with restoration of the metaphysis have been developed to optimize the results of revision TKA [4-8]. In this editorial, it was our aim to present contemporary management solutions for severe bone loss encountered at the time of revision TKA.

The main goal of revision TKA is to restore patient function with a stable, painless, and mobile knee. As such, the surgeon must overcome three basic challenges: (1) implant fixation, (2) alignment in all planes, and (3) proper patellofemoral tracking. The unifying principle that often limits achieving these goals is marked bone loss. In modern revision TKA systems, stems and augments are very helpful. However, the main challenge remains long-lasting stability of the implants despite a significant bone loss [4].

Two types of bone defects can be observed in revision TKA: cavitary defects or segmental defects [4]. Classifications [such as the American Academy of Orthopaedic Surgeons (AAOS) and Anderson Orthopaedic Research Institute (AORI) classification] have been created to categorize the bone loss, which may be difficult to determine preoperatively, particularly when implants must be first removed [4]. In reality, preoperative radiographic assessment helps determine whether the defect is segmental, cavitary, or combined [4]. This is important because segmental defects should be re-constructed to properly support the implant. In addition, the level of constraint should be adapted accordingly if the bony insertion of the collateral ligaments is not present or functional [4].

Unfortunately, utilizing undersized press-fit stems and adding more bone cement are still utilized to manage many large bony defects [4]. Morcellized allograft still has a role 
for small cavitary defects, while bulk allograft has a limited role for segmental defects. The limitations of bulk allograft include mid-term failures due to a lack of osseous integration [4]. Augments are very helpful for limited segmental defects. On the other hand, sleeves allow for metaphyseal restoration [4]. However, the current tribology of metaphyseal sleeves limits bony ingrowth [4]. In addition, both augments and sleeves require long, uncemented diaphyseal stems that are often undersized [4]. Those that are appropriately sized often lead to end of stem pain.

Many of the above limitations have been obviated with highly porous tantalum cones [5-8]. Such cones have a host of benefits, including metaphyseal restoration, biologic fixation, and the ability to utilize shorter, cemented stems for immediate axial stability and rotational rigid fixation [5-8]. The key is tantalum, element 73 on the periodic table [5-8]. Tantalum is vapor deposited onto a pyrolytic carbon scaffold creating a three-dimensional structure with interconnected porosity of approximately $70 \%$ [5-8]. The high porosity and high surface roughness appears to be important to stimulate bony ingrowth [5-8]. Results of animal pre-clinical studies have shown that Trabecular Metal ${ }^{\circledR}$ is completely integrated after 24 weeks [5-8]. Mechanical properties of the tantalum are also very important [5-8]. First, tantalum has a very high coefficient of friction, resulting in excellent primary stability upon impaction. Second, the modulus of elasticity of tantalum is similar to cancellous bone [5-8]. As such, the mechanical support is strong enough to support the implant without destroying the surrounding bone. Practically speaking, the external portion of the cone should be against the host bone and inserted in a press-fit manner [5-8]. When the contact is not optimal due to irregularity of the host bone surface, a bone substitute can be added. This addition of a bone substitute is important to create a biological structure including the cone to host bone. This construct restores a biological support at the metaphyseal aspect of the tibia or at the distal part of the femur. The implants with short stems are then cemented into this construct.

After the initial description published by the Mayo team, series with 2-5 years of follow-up are now reported in the literature [6]. Short and mid-term results recently published in the literature show very promising results with high functional improvements and good survivorship [8]. Our series with a 2- to 6-year follow-up compares favorably with the results of the literature, with good stability of the implants and satisfying functional results [5-8]. The minimum 10-year results of these techniques will be interesting and mandatory to show an improvement at longer-term follow-up compared to other techniques such as allografts.

The cones are now widely used both in North America and in Europe. In the very near future, we anticipate that such techniques will be the standard of care for management of major bone loss in revision TKA, with most major companies providing a similar product. In the future, one area of interest will be utilizing the highly porous tantalum as a delivery vehicle through physician-directed applications.

\section{References}

1. Dalury DF, Pomeroy DL, Gorab RS, Adams MJ (2013) Why are total knee arthroplasties being revised. J Arthroplasty $28(8$ Suppl.):120-121

2. Bhandari M, Smith J, Miller LE, Block JE (2012) Clinical and economic burden of revision knee arthroplasty. Clin Med Insights Arthritis Musculoskelet Disord 5:89-94

3. Pfefferle KJ, Gil KM, Fening SD, Dilisio MF (2014) Validation study of a pooled electronic healthcare database: the effect of obesity on the revision rate of total knee arthroplasty. Eur J Orthop Surg Traumatol 24(8):1625-1628

4. Huten D (2013) Femorotibial bone loss during revision total knee arthroplasty. Orthop Traumatol Surg Res 99(1 Suppl.):22-33

5. Long WJ, Scuderi GR (2009) Porous tantalum cones for large metaphyseal tibial defects in revision total knee arthroplasty: a minimum 2-year follow-up. J Arthroplasty 24(7):1086-1092

6. Meneghini RM, Lewallen DG, Hanssen AD (2008) Use of porous tantalum metaphyseal cones for severe tibial bone loss during revision total knee replacement. J Bone Joint Surg Am 90(1):78-84

7. Jensen CL, Petersen MM, Schrøder HM, Flivik G, Lund B (2012) Revision total knee arthroplasty with the use of trabecular metal cones: a randomized radiostereometric analysis with 2 years of follow-up. J Arthroplasty 27(10):1820-1826

8. Kamath AF, Lewallen DG, Hanssen AD (2015) Porous tantalum metaphyseal cones for severe tibial bone loss in revision knee arthroplasty: a five to nine-year follow-up. J Bone Joint Surg Am 97(3):216-223 